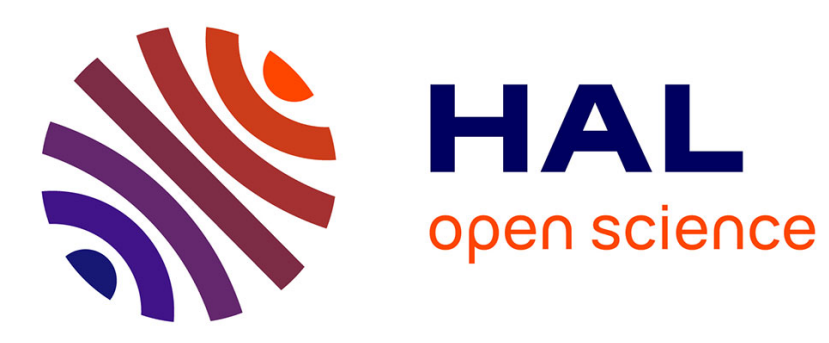

\title{
Static Modeling of Sagging Cables With Flexural Rigidity and Shear Forces
}

\author{
Hussein Hussein, Marc Gouttefarde, François Pierrot
}

\section{To cite this version:}

Hussein Hussein, Marc Gouttefarde, François Pierrot. Static Modeling of Sagging Cables With Flexural Rigidity and Shear Forces. ARK 2018 - 16th International Symposium on Advances in Robot Kinematics, Jul 2018, Bologna, Italy. pp.310-318, 10.1007/978-3-319-93188-3_36 . lirmm-01897992

\section{HAL Id: lirmm-01897992 https://hal-lirmm.ccsd.cnrs.fr/lirmm-01897992}

Submitted on 17 Oct 2018

HAL is a multi-disciplinary open access archive for the deposit and dissemination of scientific research documents, whether they are published or not. The documents may come from teaching and research institutions in France or abroad, or from public or private research centers.
L'archive ouverte pluridisciplinaire HAL, est destinée au dépôt et à la diffusion de documents scientifiques de niveau recherche, publiés ou non, émanant des établissements d'enseignement et de recherche français ou étrangers, des laboratoires publics ou privés. 


\title{
Static Modeling of Sagging Cables With Flexural Rigidity and Shear Forces
}

\author{
Hussein Hussein, Marc Gouttefarde, and François Pierrot \\ LIRMM, Université de Montpellier, CNRS, Montpellier, France \\ hussein.hussein@lirmm.fr
}

\begin{abstract}
Cables are considered perfectly flexible in the classic modeling without flexural rigidity and with purely tangential cable forces. In this paper, a static modeling of cables is presented where flexural rigidity and shear forces are considered. The calculation details are presented with and without considering the axial extensibility of cables for three problems where one of the parameters including cable length, end point positions and forces is determined by knowing the two others. The effects of considering flexural rigidity and shear forces are then analyzed on a simple example of a cable-driven parallel robot consisting of a point mass attached to two cables.
\end{abstract}

Keywords: Cable modeling, flexural rigidity and shear forces, cabledriven parallel robots

\section{Introduction}

In several previous works, e.g. [3, 6, 8, 14], the static analysis of large-dimension cable-driven parallel robots has been studied using the well-known elastic catenary cable modeling [7] in order to account for the cable mass and elasticity. The resulting kinetostatic modeling consists of a set of nonlinear equations involving the cable lengths, the forces applied by the cables on the robot mobile platform, and the mobile platform pose. Due to their nonlinear nature, numerical methods must be considered to solve the corresponding inverse and direct kinetostatic problems $[8,11,12]$. These methods might be relatively slow because of the involved computations which may be impractical in real-time environments. Hence, simplifications of this kinetostatic modeling have been considered in $[5,10,13]$ where assumptions on the cable model are made, e.g. considering that the cable elasticity has a little influence on the cable shape. In fact, the elastic catenary cable model [7] is also based on some assumptions, one of them being to consider that the cable has a negligible flexural rigidity.

The contribution of the present paper is thus a formulation of the static modeling of cables of non-negligible mass, where flexural rigidity and shear forces are taken into account. Three problems are considered in the modeling where one of the parameters including cable length, end point positions and forces is determined by knowing the two others. A simple application to a cable-driven parallel robot consisting of a point mass attached to two cables is also presented. 
In contrast to other works such as [1, 2, 9], the cable modeling in the present paper is an extension of the elastic catenary cable modeling [7] and is notably based on the Euler-Bernoulli bending moment expression for large deformations. This modeling aims at investigating the effects of considering bending moments on the accuracy of end point positioning, and on the determination of cable length and forces, in order to be used for cable-driven parallel robots.

The paper is organized as follows. In Section 2, the static modeling of an elastic cable with consideration of flexural rigidity and shear forces is presented. Based on this modeling, Section 3 discusses three problems where the cable length, end point positions or forces is to be determined when the two others are known. Finally, in Section 4, an example application of a point mass attached to two cables is presented.

\section{Cable model}

The static modeling of an elastic cable with consideration of flexural rigidity and shear forces is introduced in this section. Figure 1 shows a schematic of a cable of length $L$ hanged between two points A and B which are not necessarily at the same level. The forces and moments applied on the cable and on a segment of the cable of length $p$ are shown in Fig. 1.

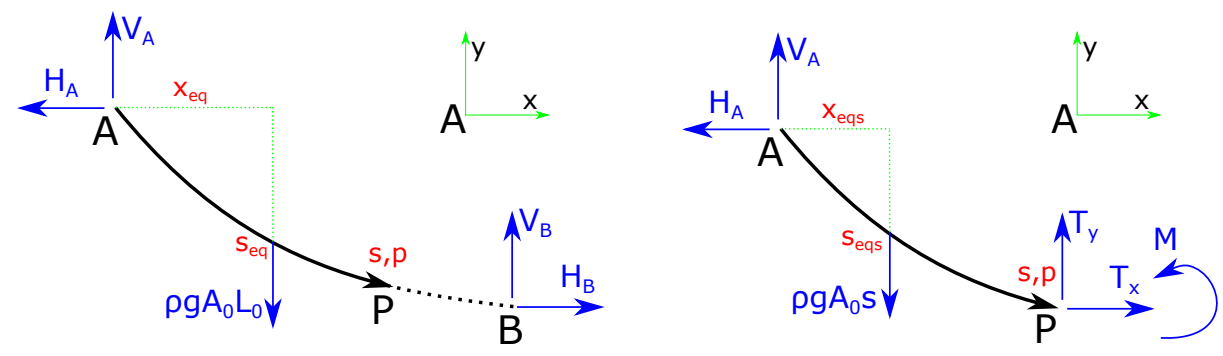

Fig. 1. Schematic of the cable with the different notations.

No moment is considered to be applied on the cable end points which is generally the case in cable robot applications. The axes $\mathrm{x}$ and $\mathrm{y}$ are the horizontal and vertical axes, respectively. The point $A$ is taken as the origin of the $x-y$ frame. The cable profile is denoted by $w$ (y coordinate). The strained length of the cable segment between point $A$ and point $P$ is denoted by $p$ while its unstrained length is denoted by $s$. The coordinates of point $P$ are $(x, w)$. The relationship between $x, w$ and $p$ is defined by the following geometric constraint:

$$
d p^{2}=d x^{2}+d w^{2}
$$

The force and moment static equilibrium equations of the cable segment $A P$ are: 


$$
\left\{\begin{array}{l}
T_{x}=H_{A} \\
T_{z}=\rho g A_{0} s-V_{A} \\
M=V_{A} x+H_{A} w-\rho g A_{0} s\left(x-x_{e q s}\right)
\end{array}\right.
$$

where $g$ is the gravity acceleration, $\rho$ the cable density, $A_{0}$ the unstrained cable cross-section area, and $H_{A}$ and $V_{A}$ are the horizontal and vertical cable force components at point $A$, respectively. The force components $\left(H_{B}, V_{B}\right)$ at point $B$ can be determined using the first two equations in (2), with $s=L$, once the cable length and force components at point $A$ are determined. Thus, only the force components at point $A, H_{A}$ and $V_{A}$, are considered in the sequel. Note that $\rho g A_{0} s$ is the weight of the cable segment $A P$. It is written using the unstrained length $s$ since the known parameters are generally the unstrained cable length $L$ and the unstrained cross-section area $A_{0}$.

As illustrated in Fig. 1, $x_{\text {eqs }}$ in (2) is the position along $\mathrm{x}$ of the center of mass of the cable segment $[0, s] . x_{\text {eqs }}$ is given by:

$$
x_{e q s}=\frac{1}{s} \int_{0}^{s} x d s
$$

Considering axial elasticity, $s$ and $p$ are related by Hooke's law as follows:

$$
\frac{d p}{d s}=1+\frac{T_{\alpha}}{E A_{0}}
$$

where $E$ is the Young's modulus and $T_{\alpha}$ is the tangential component of the cable force defined as:

$$
T_{\alpha}=T_{x} \cos \alpha+T_{z} \sin \alpha=H_{A} \cos \alpha+\left(\rho g A s-V_{A}\right) \sin \alpha
$$

where $\alpha$ is the slope angle of the cable along its length.

The classic sagging cable model (elastic catenary) used in civil engineering since the 1930s, takes the cable mass and axial elasticity into account. It is based on the equations presented above and will be referred to as the "classic model" in the sequel. The mathematical details of this model are presented e.g. in $[7,8]$. The cable in the classic model is assumed to be perfectly flexible, devoid of flexural rigidity (bending moments are neglected). The action of any part of the cable upon its neighbor is purely tangential. The static cable profile is then defined by a set of non-linear equations:

$$
\left\{\begin{array}{l}
x=\frac{H_{A}}{E A_{0}} s+\frac{H_{A}}{\rho g A_{0}} \ln \frac{\sqrt{H_{A}^{2}+V_{A}^{2}}+V_{A}}{\sqrt{H_{A}^{2}+\left(V_{A}-\rho g A_{0} s\right)^{2}}+V_{A}-\rho g A_{0} s} \\
w=\frac{s}{E A_{0}}\left(\frac{\rho g A_{0} s}{2}-V_{A}\right)+\frac{1}{\rho g A_{0}}\left(\sqrt{H_{A}^{2}+\left(V_{A}-\rho g A_{0} s\right)^{2}}-\sqrt{H_{A}^{2}+V_{A}^{2}}\right)
\end{array}\right.
$$

The modeling presented in this paper does not consider the assumptions of the classic model and investigates the effects of the bending moments on the statics of the cable. The Euler-Bernoulli law states that the bending moment $M$ is proportional to the change in the curvature produced by the action of the load [4]. The bending moment expression according to this law is: 


$$
M=E I \frac{\frac{d^{2} w}{d x^{2}}}{\left(1+\left(\frac{d w}{d x}\right)^{2}\right)^{\frac{3}{2}}}
$$

where $I$ is the second moment of area of the cable cross-section. For simplification, the following variable substitution can be made:

$$
\left\{\begin{array}{l}
d x=d p \cos \alpha \\
d w=d p \sin \alpha
\end{array}\right.
$$

Considering the new variable $\alpha$, and substituting (7) and (3) in the third equation of (2), the governing equation of the cable is obtained as:

$$
E I \frac{d \alpha}{d p}=V_{A} x+H_{A} w-\rho g A_{0} s x+\rho g A_{0} \int_{0}^{s} x d s
$$

where the following equation has been used:

$$
\frac{d \alpha}{d p}=\frac{\frac{d^{2} w}{d x^{2}}}{\left(1+\left(\frac{d w}{d x}\right)^{2}\right)^{\frac{3}{2}}}
$$

The differentiation in (9) must be made with respect to the unstrained length $s$ when the boundary conditions are expressed in $s$. The relation between $d p$ and $d s$ is obtained from (4). This relation is dependent of the tangential cable tension $T_{\alpha}$.

The cable force being assumed to be tangent to the cable profile in the classic model, the right-hand side of (9) can be shown to be zero [7]. Then, according

to (10), $\frac{d^{2} w}{d x^{2}}=0$ which means that the cable is a straight line and, thus, without sagging. It does not correspond to the real case since the cable mass is considered in the modeling. To be rigorous, the bending moment should thus be taken into account and shear forces must also be considered since the cable force is not necessarily tangent to the cable profile.

Let us note that the moment expression with small deformations, $M=$ $E I \frac{d^{2} w}{d x^{2}}$ in $(7)$, cannot be used in the case of inclined cables since the cable slope $\frac{d w}{d x}$ cannot be neglected. One solution for taut cables is to use an inclined orthogonal frame $\left(x^{\prime}, y^{\prime}\right)$ where $x^{\prime}$ is parallel to the line AB. In this case, the governing equation obtained from the equilibrium is simplified with the small deformation expression and can be solved using Fourier series. This solution is not shown in this paper since the more general case with large deformation expression of the moment is treated.

\section{Modeling problems and solutions}

Generally, in cable robot applications, the position of the end point $A$ is assumed to be known, and the main parameters to be calculated are the cable unstrained 
length $L$, the position of the cable end point $B$, and the force components at the cable end points. Three main problems can then be defined. In the first one, the length and forces are known and the end point position must be determined. In the second one, the length and end point position are known and the forces have to be calculated. In the third one, the end point position and forces are known and the unknown is the cable length. With the cable modeling introduced in Section 2, a solution method is presented for each of these problems in the following subsections.

\subsection{First problem: Knowing length and forces and searching for position}

The differential equation in (9) governs the static behavior of the cable. Because of the nonlinearity of the equation, explicit expressions for $x$ and $w$ cannot be obtained, contrary to the case of the classic model.

Hence, an ODE must be considered and the first, second, and third problems defined above are boundary value problems that can be solved using numerical methods. The solvers 'bvp4c' or 'bvp5c', which are finite difference codes available in Matlab, have been used. Accordingly, a system of differential equations of the first order must be written and the number of boundary conditions must be equal to the number of equations in this system.

In order to solve the first problem, the ODE (9) is differentiated with respect to $s$ and written as a system of five first order differential equations $\left(\boldsymbol{y}^{\prime}=\right.$ $\left.f_{1}(s, \boldsymbol{y})\right)$ with five variables $\left(\boldsymbol{y}=\left[x, w, \alpha, \frac{d \alpha}{d s}, \int_{0}^{s} x d s\right]\right)$. When the axial elasticity (extensibility) is not considered, the system of first order differential equations is simplified by considering that $s$ and $p$ are equal and $d s / d p$ is equal to 1 . Five boundary conditions are introduced in order to solve the problem, which are related to the position of the cable end point $A(x(0)=0, w(0)=0)$, to the value of $\int_{0}^{s} x d s$ at $A\left(\int_{0}^{s} x d s(0)=0\right)$, and to the moments at $A$ and $B$ $(M(0)=M(L)=0)$.

\subsection{Second problem: Knowing length and position and searching for forces}

When searching for the forces in the case of the second problem, the force components $H_{A}$ and $V_{A}$ are unknown in the governing ODE (9) while two additional boundary conditions related to the position of the cable end point $B$ are added.

The solvers 'bvp4c' and 'bvp5c' allow solving ODE with additional unknown parameters by adding more boundary conditions. Taking advantage of this possibility, using the same system of first order differential equations defined for the first problem, the second problem can be solved by considering $H_{A}$ and $V_{A}$ as unknowns. Seven boundary conditions are introduced in this case in order to solve the problem. These conditions are related to the position of the cable end points $A(x(0)=0, w(0)=0)$ and $B\left(x(L)=x_{B}, w(L)=y_{B}\right)$, to the value of $\int_{0}^{s} x d s$ at $A\left(\int_{0}^{s} x d s(0)=0\right)$, and to the moments at $A$ and $B(M(0)=M(L)=0)$. 


\subsection{Third problem: Knowing position and forces and searching for length}

In the case of the third problem, the length is unknown which requires to change the differentiation variable in the governing ODE. In the first two cases, differentiation with respect to $s$ is possible since the bounds on $\mathrm{s}(s \in[0, L])$ are well defined, which is not the case in the third problem.

The other possible differentiation variables are $x$ and $w$. Differentiation with respect to $x$ is considered since $x$ is a monotonic function of the cable length $s$ or $p$, which is not the case for $w$. Therefore, another variable substitution is made as follows:

$$
\left\{\begin{array}{l}
d x=d p \cdot \sqrt{1-u^{2}} \\
d w=d p \cdot u
\end{array}\right.
$$

where $u$ is defined by (11), the latter being consistent with (1).

Considering the new variable substitution with $u$, the ODE in (9) becomes:

$$
E I \frac{d u}{d x}=V_{A} x+H_{A} w-\rho g A_{0} \int_{0}^{s} s d x
$$

Differentiating with respect to $x$ one time, the following ODE is obtained:

$$
\frac{d^{2} u}{d x^{2}}=V_{A}+\frac{H_{A}}{E I} \frac{u}{\sqrt{1-u^{2}}}-\frac{\rho g A_{0}}{E I} s
$$

For a given position of $B$, note that the cable tension components $H_{A}$ and $V_{A}$ are dependent on each other. Consequently, only one force component $H_{A}$ or $V_{A}$ can be defined. This is obvious in the case of the inextensible cable model since the differentiation of (13) with respect to $x$ eliminates $V_{A}$ so that the problem can be solved without considering it.

Moreover, considering that the cable tension $T_{A}=\sqrt{H_{A}^{2}+V_{A}^{2}}$ can be measured using a force sensor, it is considered that $T_{A}$ is known in the third problem.

Besides, knowing that $H_{A}$ is always positive, it can be replaced by $H_{A}=$ $\sqrt{T_{A}^{2}-V_{A}^{2}}$ in the equations. The governing ODE (13) becomes:

$$
\frac{d^{2} u}{d x^{2}}=V_{A}+\frac{\sqrt{T_{A}^{2}-V_{A}^{2}}}{E I} \frac{u}{\sqrt{1-u^{2}}}-\frac{\rho g A_{0}}{E I} s
$$

Thereby, the system of first order equations defining the third problem $\left(\boldsymbol{y}^{\prime}=\right.$ $\left.f_{2}(x, \boldsymbol{y})\right)$ has five variables $\left(\boldsymbol{y}=\left[w, s, \int_{0}^{x} s d x, u, \frac{d u}{d x}\right]\right)$, one unknown $V_{A}$ and six boundary conditions. These conditions are related to the position of the cable end points $A(w(0)=0, s(0)=0)$ and $B(w(L)=0)$, to the value of $\int_{0}^{x} s d x$ at $A\left(\int_{0}^{x} s d x(0)=0\right)$, and to the moments at $A$ and $B(M(0)=M(L)=0)$.

\section{Application: Two cables attached to a point mass}

In this section, the extensible and inextensible cable models introduced in the previous sections (referred to as the new models in the sequel) are compared 


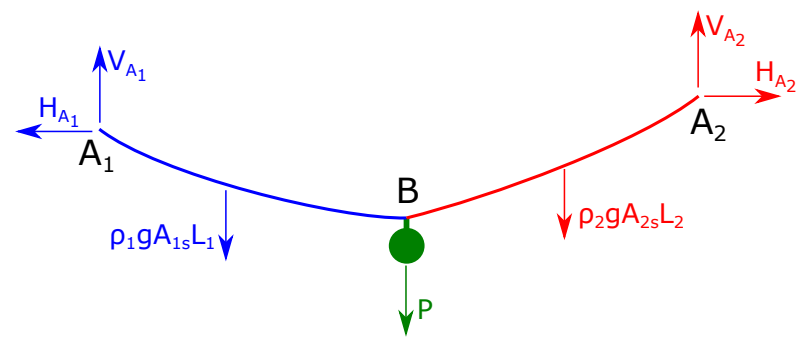

Fig. 2. Schematic of two cables attached to a point mass B.

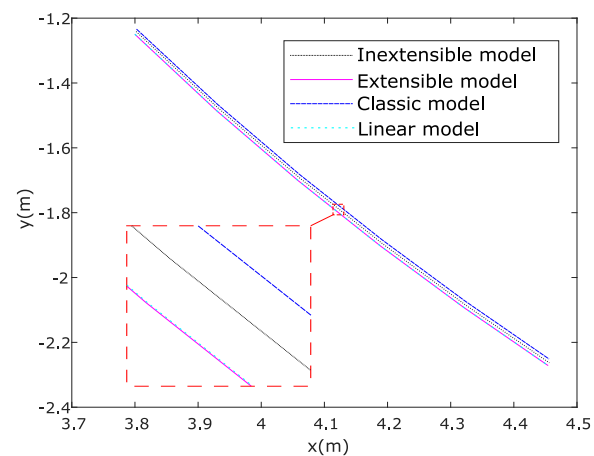

(a)

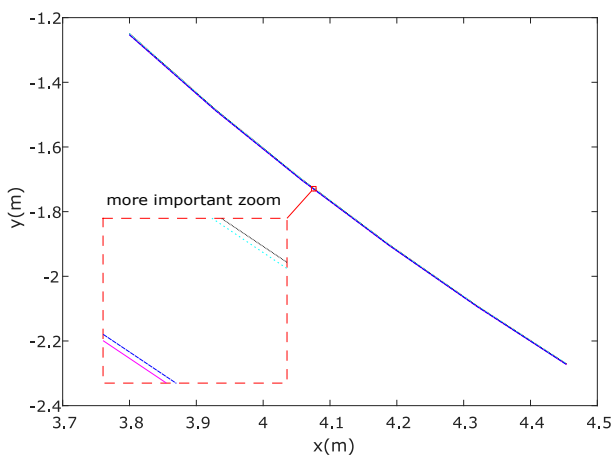

(b)

Fig. 3. Trajectories obtained with the different models for a weight $\mathrm{P}=0.5 \mathrm{kN}$ (a) and $\mathrm{P}=10 \mathrm{KN}(\mathrm{b})$.

with the classic cable model (elastic catenary). To this end, as a simple example of a cable-driven parallel robot, the trajectory of a point mass attached to two cables is considered (Fig. 2). Changing the length of one cable, the trajectory of the point mass is calculated with the new and classic models as well as with a linear model where no sagging is considered.

The cables have a radius of $1 \mathrm{~cm}$, density $8050 \mathrm{~kg} / \mathrm{m}^{3}$ and Young's modulus $170 \mathrm{GPa}$. The coordinates of $A_{1}$ and $A_{2}$ are defined as $(0 ; 0) \mathrm{m}$ and $(10 ; 2) \mathrm{m}$, respectively. The unstrained length $L_{2}$ of the second cable is fixed at $7 \mathrm{~m}$ while the length $L_{1}$ of the first cable is varied between $4 \mathrm{~m}$ and $5 \mathrm{~m}$. Figure 3 shows the trajectory obtained with the different models and with two weights $P=0.5 k N$ and $P=10 k N$ of the point mass.

The trajectory calculated with the linear model is independent of the weight $P$. Figure 3 shows that the trajectory with the new extensible model is close to the linear model for a moderate weight $P$. The rationale of this rather surprising result requires further investigation. In addition, for a moderate weight $P$, the trajectories obtained with the new inextensible model and with the classic model are farther away. Besides, we notice that the trajectories get closer to the linear model when the weight is increased. This can be expected since the effect of the 
cable sagging is lower for higher tensions. In effect, the trajectories for a heavy weight become much closer to each other.

Besides, as shown in the zoom in Fig. 3.b, the trajectory of the new inextensible model becomes much closer to the trajectory of the linear model. However, the trajectories of the new extensible model and classic model are much closer to each other at a lower level along the vertical $y$ axis which is due to the effect of the axial elongation of the cables.

Note that the calculation of the trajectory with the new models is not possible using directly 'bvp4c' or 'bvp5c' solvers since the governing equations of the two cables are coupled by the static equilibrium of the point mass and these solvers do not solve multi-region boundary value problems. The trajectories shown in Fig. 3 were calculated at each point of a discretization of the trajectory by sweeping the unknown parameters so as to satisfy the equilibrium equations. The corresponding calculations are time consuming. One future work on the modeling presented in this paper is thus to reduce this computation time, which is required if a cable-driven parallel robot driven by six or more cables is considered. It should be possible either by using previously calculated data in online calculations or by finding an algorithm to solve directly the coupled multi-region boundary value problem.

\section{Conclusion}

A static modeling of cables was presented in this paper. It takes into account the cable flexural rigidity which is not considered in the usual elastic catenary model. The shear forces are thereby not neglected and the cable force is not necessarily tangent to the sagging cable profile. The calculation details were presented with and without considering extensibility for three main problems where one of the parameters among cable length, end point positions and forces is determined by knowing the two others. A comparison with the elastic catenary cable model was made in a simple application of a cable-driven parallel robot consisting of a point mass attached to two cables. Future works include comparisons to experimental data and computation efficiency improvements in the case of an application to cable-driven parallel robots.

\section{Acknowledgements}

The research leading to these results has received funding from the European Union's H2020 Programme (H2020/2014-2020) under grant agreement No. 732513. 


\section{Bibliography}

[1] Boyer, F., De Nayer, G., Leroyer, A., Visonneau, M.: Geometrically exact kirchhoff beam theory: application to cable dynamics. Journal of Computational and Nonlinear Dynamics 6(4), 041,004 (2011)

[2] Caballero, A., Poser, M.: Local bending stresses in stay cables with an elastic guide. Structural Engineering International 20(3), 254-259 (2010)

[3] Du, J., Bao, H., Duan, X., Cui, C.: Jacobian analysis of a long-span cabledriven manipulator and its application to forward solution. Mechanism and Machine Theory 45, 1227-1238 (2010)

[4] Fertis, D.G.: Basic theories and principles of nonlinear beam deformations. Nonlinear Structural Engineering: With Unique Theories and Methods to Solve Effectively Complex Nonlinear Problems pp. 1-61 (2006)

[5] Gouttefarde, M., Collard, J.F., Riehl, N., Baradat, C.: Simplified static analysis of large-dimension parallel cable-driven robots. In: Proc. IEEE Int. Conf. Robotics and Automation (ICRA), pp. 2299-2305 (2012)

[6] Gouttefarde, M., Nguyen, D.Q., Baradat, C.: Kinetostatic analysis of cabledriven parallel robots with consideration of sagging and pulleys. In: Advances in Robot Kinematics, pp. 213-221. Springer (2014)

[7] Irvine, M.: Cable Structures. MIT Press (1981)

[8] Kozak, K., Zhou, Q., Wang, J.: Static analysis of cable-driven manipulators with non-negligible cable mass. IEEE Trans. on Robotics 22(3), 425-433 (2006)

[9] Lang, H., Linn, J., Arnold, M.: Multi-body dynamics simulation of geometrically exact cosserat rods. Multibody System Dynamics 25(3), 285-312 (2011)

[10] Li, H., Zhang, X., Yao, R., Sun, J., Pan, G., Zhu, W.: Optimal force distribution based on slack rope model in the incompletely constrained cable-driven parallel mechanism of FAST telescope. In: T. Bruckmann, A. Pott (eds.) Cable-Driven Parallel Robots, pp. 87-102. Springer (2013)

[11] Merlet, J.P.: The kinematics of cable-driven parallel robots with sagging cables: preliminary results. In: Proc. IEEE Int. Conf. Robotics and Automation (ICRA), pp. 1593-1598. Seattle, Washington (2015)

[12] Merlet, J.P.: A new generic approach for the inverse kinematics of cabledriven parallel robots with 6 deformable cables. In: Advances in Robot Kinematics (ARK) (2016)

[13] Nguyen, D.Q., Gouttefarde, M., Company, O., Pierrot, F.: On the simplifications of cable model in static analysis of large-dimension cable-driven parallel robots. In: Proc. IEEE/RSJ Int. Conf. Intelligent Robots and Systems (IROS), pp. 928-934. Tokyo, Japan (2013)

[14] Ottaviano, E., Castelli, G.: A study on the effects of cable mass and elasticity in cable-based parallel manipulator. Proc. of the 18th CISM-IFToMM Symp. On Robot Design, Dynamics and Control, Springer Ed. Udine pp. 149-156 (2010) 